\title{
Investigation on the Working Point of Slope-Assisted Dynamic Brillouin Distributed Fiber Sensing
}

\author{
Cheng Feng ${ }^{\mathrm{a}}$, Jon Mariñelarena ${ }^{\mathrm{b}}$, and Thomas Schneider ${ }^{\mathrm{a}}$ \\ ${ }^{a} \mathrm{THz}$ Photonics Group, Technische Universität Braunschweig, 38106 Braunschweig, Germany \\ ${ }^{\mathrm{b}}$ Department of Electrical and Electronic Engineering, Universidad Pública de Navarra, \\ Campus Arrosadia s/n, 31006 Pamplona, Spain
}

\begin{abstract}
In this paper, an investigation on the working point of slope-assisted dynamic distributed Brillouin sensing is presented. A comparison has been carried out between the sensing performances achieved at the inflection point and the $3 \mathrm{~dB}$ point of the Brillouin gain spectrum. Besides the intrinsic 13.1\% frequency-to-amplitude sensitivity enhancement and a higher signal level, the dynamic sensing at the inflection point can achieve a doubled in maximum and in average a $36.8 \%$ wider dynamic range with much better working point symmetry. Simulations with strain signals also demonstrate that, compared to the $3 \mathrm{~dB}$ point, the average error at the inflection point can be significantly reduced to only $27.7 \%$. As shown in this work, by a simple shift of the working point from the $3 \mathrm{~dB}$ to the inflection point, slope-assisted dynamic sensing can be well enhanced.
\end{abstract}

Keywords: Stimulated Brillouin scattering, Brillouin optical time-domain analyzer, dynamic sensing, slopeassisted, inflection point

\section{INTRODUCTION}

Distributed fiber sensors (DFS) have been intensively investigated due to their unique ability to measure the temperature and strain distribution with a high spatial resolution. The Brillouin optical time-domain analyzer (BOTDA), as the simplest DFS based on stimulated Brillouin scattering (SBS), has attracted enormous interests since its first proposal. ${ }^{1}$ Its principle can be classically described as an interaction between a pulsed pump wave and a counter-propagating, frequency down-shifted probe continuous wave via an acoustic wave. In comparison with other DFS techniques, BOTDA owns not only excellent sensing performances, such as long sensing range and high spatial and measurand (temperature or strain) accuracy, ${ }^{2}$ but also benefits from striking advantages of SBS, such as low threshold and high gain. However, unlike an CW-SBS interaction, the Brillouin gain of the BOTDA is usually limited by the interaction length. In order to maintain the high accuracy, a large amount of averaging needs to be applied on the measured signal for a better signal-to-noise ratio (SNR). ${ }^{3}$ Furthermore, in order to estimate the measurand dependent Brillouin frequency shift (BFS), the scanning over the whole Brillouin gain spectrum (BGS) has to be carried out. ${ }^{2}$ Despite the intrinsic narrow BGS linewidth of 20-30 MHz, which can be further narrowed by one order of magnitude, ${ }^{4-7}$ it still takes up to several minutes to carry out the full scanning and averaging. Such a long measurement time makes a conventional BOTDA not applicable for dynamic tasks.

In the past decades, several techniques have been proposed for dynamic BOTDA sensing. On one hand, the SNR can be enhanced by self-heterodyne detection ${ }^{8}$ and coding. ${ }^{9}$ Therefore, the requirement on the number of averaging can be reduced with a sufficient SNR. On the other hand, various kinds of multi-tone interrogation ${ }^{10,11}$ and fast frequency scanning ${ }^{3}$ methods enable the BGS acquirement with a single-shot (or negligible time). Nevertheless, these methods either complicate the setup or have a very high demand on the devices. The most simple and applicable BOTDA dynamic sensing technique, is slope-assisted (SA) sensing. ${ }^{12}$ The full BGS scanning is avoided by the frequency-to-amplitude sensitivity at the BGS edge. Considering that the BGS linewidth can be controlled by the pump linewidth, ${ }^{13}$ several methods can be applied to engineer the dynamic

Further author information: (Send correspondence to C.F.)

C.F.: E-mail: cheng.feng@ihf.tu-bs.de, Telephone: +49 (0)531 391-2011 


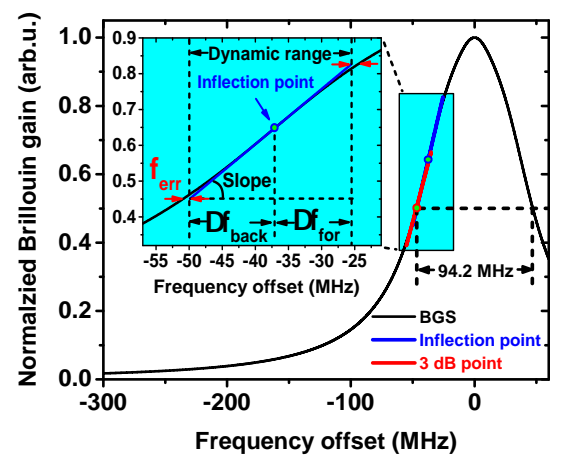

Figure 1. Normalized and fitted BGS generated by a $10 \mathrm{~ns}$ pump pulse (black) and the typical dynamic ranges achieved at inflection (blue) and $3 \mathrm{~dB}$ point (red). (Inset: Definition of the dynamic range, sensitivity and the symmetry factor)

range. ${ }^{14}$ However, since the first propose of this technique, ${ }^{12}$ the working point is arbitrarily set at the $3 \mathrm{~dB}$ point of the BGS, with no specified reasons or comparisons of sensing performances at other working points.

In this paper, a detailed investigation on the working point of the SA-BOTDA is given under consideration of sensitivity, dynamic range, working point symmetry and measurement (frequency) error. The results indicate that, working at the inflection point of the BGS brings not only $13.1 \%$ sensitivity enhancement, but also a doubled at maximum and in average $36.8 \%$ wider dynamic range with much better working point symmetry than at the $3 \mathrm{~dB}$ point. The error of a retrieved strain signal at the inflection point can also be reduced to only $27.7 \%$ of the one at the $3 \mathrm{~dB}$ point. Thus, in comparison with the $3 \mathrm{~dB}$ point, the inflection point is more ideal for SA-dynamic sensing.

\section{PRINCIPLE}

The principle of SA-BOTDA dynamic sensing is based on the linear dependence of the strain on the BFS via the strain coefficient $C_{\varepsilon}=50 \mathrm{kHz} / \mu \varepsilon^{2}$ and the quasi-linear frequency-to-amplitude sensitivity on the BGS edge. Therefore, the frequency shift can be approximated according to the Taylor expansion as:

$$
\Delta f \approx\left[g\left(f_{c}+\Delta f\right)-g\left(f_{c}\right)\right] / S\left(f_{c}\right)
$$

where $g$ is the Brillouin gain, $f_{c}$ is the working point frequency, $\Delta f$ is the frequency shift to be measured and $S\left(f_{c}\right)$ is the sensitivity at the frequency $f_{c}$. The frequency shift estimation from Equation (1) only works well, if the fitting error is negligible. Therefore, in order to get a good linear fit in a wider frequency range, it is better to set the working point at the inflection point of the BGS, where the derivative of $S\left(f_{c}\right)$ is negligible.

In order to verify the above mentioned theory, an experiment with the conventional BOTDA setup has been carried out. ${ }^{2}$ Considering the fiber length of a real dynamic sensing is only $\sim 100 \mathrm{~m}$, the pulse width is set to be $10 \mathrm{~ns}$ to achieve $1 \mathrm{~m}$ spatial resolution. Fig. 1 illustrates the achieved BGS with the linear fittings at the inflection and $3 \mathrm{~dB}$ point. Due to the property of the Lorentzian (and the convoluted Voigt ${ }^{14}$ ) profile, the inflection point is always closer to the BGS center with a higher gain, which makes the detection at this point easier and $13.1 \%$ more sensitive. In the inset of Fig. 1, the dynamic range is defined as the frequency span of the linear fit when the frequency error (between the linear fit and the BGS) reaches a certain cut-off value $f_{\text {err }} .{ }^{15}$ This can also be understood as the maximum error tolerance in a sensing measurement. The sensitivity is represented by the slope of the linear fit. The working point symmetry is characterized by the symmetry factor, defined as the ratio of the forward $\left(\Delta f_{f o r}\right)$ and backward $\left(\Delta f_{\text {back }}\right)$ frequency span from the working point.

\section{PERFORMANCE COMPARISON}

Figure 2(a) demonstrates the frequency errors of the linear fits at the inflection and $3 \mathrm{~dB}$ point of the BGS as a function of the frequency offset to the BGS center. The curve for the inflection point shows a flat bottom, indicating a better maintenance of the linear fit in a wider range. In Fig. 2(b), the dynamic ranges are calculated 


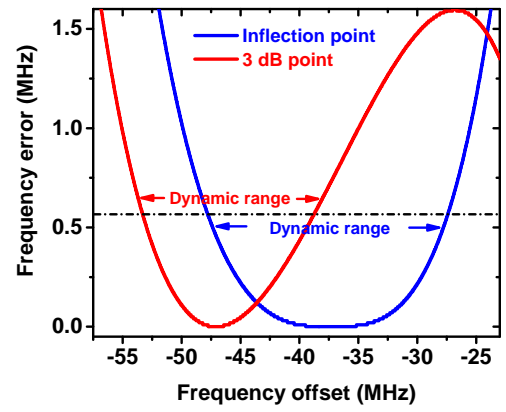

(a)

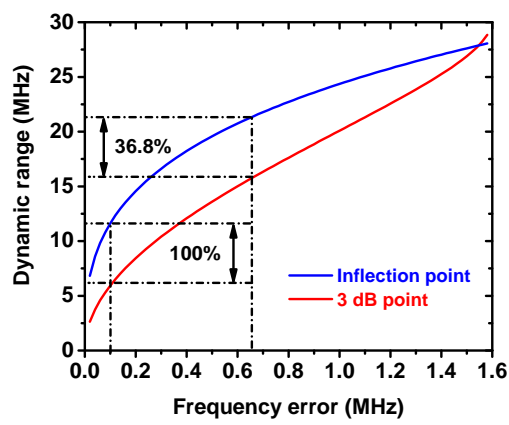

(b)

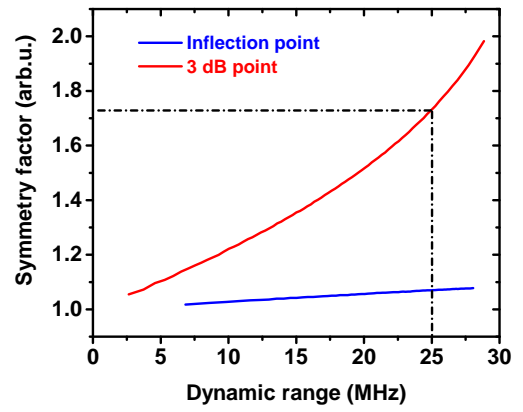

(c)

Figure 2. (a) Frequency error for the linear fits of the BGS in dependence on the frequency offset from the BGS center, (b) dynamic range under different frequency errors and (c) the symmetry factor with different dynamic ranges when working at the inflection (blue) and $3 \mathrm{~dB}$ point (red).

under various maximum frequency error tolerances. As can be seen, compared to the $3 \mathrm{~dB}$ point, the dynamic range at the inflection point is doubled in maximum and enhanced by $36.8 \%$ in average (with an error tolerance of $0.1 \mathrm{MHz}$ and $0.65 \mathrm{MHz}$, respectively). When the error tolerance exceeds $1.6 \mathrm{MHz}$, the $3 \mathrm{~dB}$ point shows a wider dynamic range than the inflection point. However, this corresponds to more than $30 \mu \varepsilon$ and up to $10 \%$ for a strain signal with an amplitude of $300 \mu \varepsilon$. Such a large error can hardly be suitable for practical applications.

Another critical issue which has to be considered is the working point symmetry. The symmetry factor is calculated and illustrated in Fig. 2(c). Note that, in order to have a better comparison, the symmetry factor is always larger than 1 . As can be clearly seen, the symmetry at the inflection point is much better than at the $3 \mathrm{~dB}$ point, indicating a much better usability of the dynamic range.

As a last step, a strain signal error analysis has been carried out by simulation. The simulated strain are $100 \mathrm{~Hz}$ sinusoidal signals with the same initial phase but different amplitudes from $150 \mu \varepsilon$ to $350 \mu \varepsilon$. The strain signals are retrieved from the detected Brillouin gain via the strain coefficient $C_{\varepsilon}$ and Equation (1), and then compared with the original strain signals. The simulation of the strain signal with the amplitude of $150 \mu \varepsilon$ is demonstrated in Visualization 1. As shown by the calculation results in Fig. 3, the strain signal is well retrieved at both points with a small amplitude $(150 \mu \varepsilon)$, but with much less error at the inflection point $(27.7 \%$ in average of the $3 \mathrm{~dB}$ point). For larger amplitudes, working at the inflection point shows also obvious advantages. Table 1 lists the specific average and maximum (strain) error (normalized to the amplitude in percent) of the strain signal simulation. Note that, though working at the $3 \mathrm{~dB}$ point might achieve relative better accuracy when the strain signal amplitude further increases (e.g. larger than $500 \mu \varepsilon$ ), the absolute error values (average and maximum error more than $8 \%$ and $21 \%$, respectively) can hardly be tolerated for practical applications.

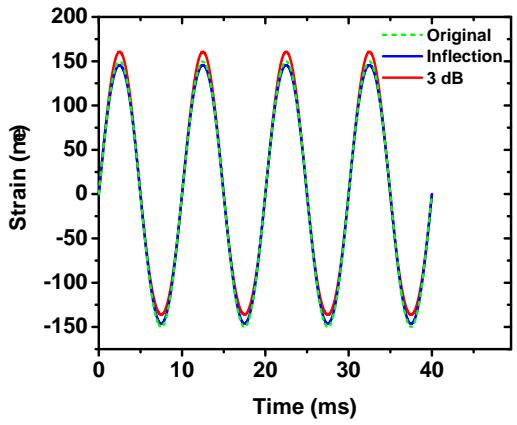

(a)

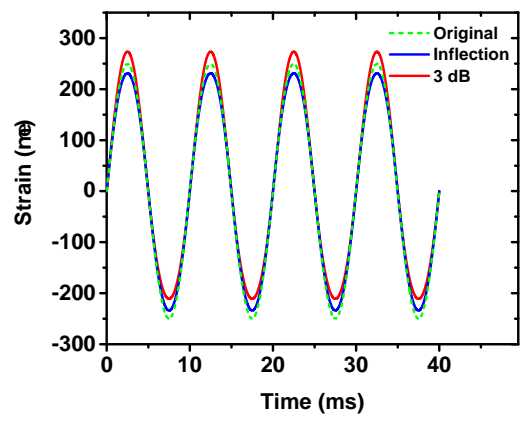

(b)

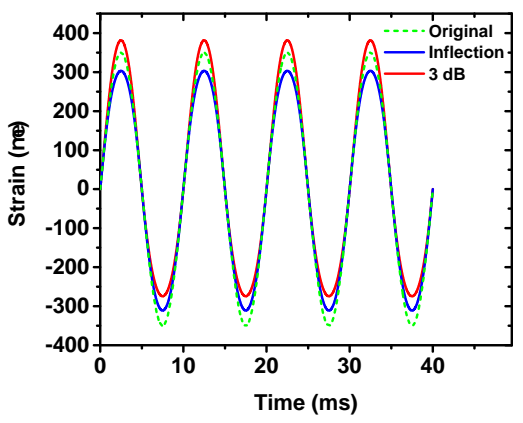

(c)

Figure 3. Dynamic strain signals (green dashed) with an amplitude of (a) $150 \mu \varepsilon$, (b) $250 \mu \varepsilon$ and (c) $350 \mu \varepsilon$ retrieved at the inflection (blue solid) and the $3 \mathrm{~dB}$ point (red solid). 
Table 1. Error analysis of the strain signal simulation.

\begin{tabular}{ccccc}
\hline Amplitude & \multicolumn{2}{c}{ Average error } & \multicolumn{2}{c}{ Maximum error } \\
\hline & inflection point & $3 \mathrm{~dB}$ point & inflection point & $3 \mathrm{~dB}$ point \\
$150 \mu \varepsilon$ & $1.15 \%$ & $4.15 \%$ & $2.89 \%$ & $9.30 \%$ \\
$250 \mu \varepsilon$ & $3.02 \%$ & $6.47 \%$ & $7.53 \%$ & $15.59 \%$ \\
$350 \mu \varepsilon$ & $5.44 \%$ & $8.20 \%$ & $13.37 \%$ & $21.60 \%$ \\
\hline
\end{tabular}

\section{CONCLUSIONS}

In this paper, the dynamic sensing performances of different working points (inflection and $3 \mathrm{~dB}$ point) of SABOTDA sensors are investigated in detail under consideration of the dynamic range, working point symmetry and strain signal error. The results show that, the inflection point of the BGS is more ideal for carrying out SA-dynamic sensing in all aspects, showing the potential to optimize the sensing technique.

\section{ACKNOWLEDGMENTS}

Cheng Feng acknowledges the financial support from the German Research Foundation (DFG SCHN 716/13-1) and Niedersächsisches Vorab (NL-4 Project "QUANOMET").

\section{REFERENCES}

[1] Horiguchi, T. and Tateda, M., "Optical-fiber-attenuation investigation using stimulated Brillouin scattering between a pulse and a continuous wave," Opt. Lett. 14(8), 408-410 (1989).

[2] Feng, C., Emad Kadum, J., and Schneider, T., "The State-of-the-Art of Brillouin Distributed Fiber Sensing," in [Brillouin Distrib. Fiber-bragg-grating-based Fiber Sens. - Princ. Meas. Appl.], IntechOpen (2019).

[3] Peled, Y., Motil, A., and Tur, M., "Fast Brillouin optical time domain analysis for dynamic sensing," Opt. Express 20(8), 8584-8591 (2012).

[4] Preussler, S., Wiatrek, A., Jamshidi, K., and Schneider, T., "Brillouin scattering gain bandwidth reduction down to 3.4MHz.," Opt. Express 19(9), 8565-8570 (2011).

[5] Preussler, S. and Schneider, T., "Bandwidth reduction in a multistage Brillouin system," Opt. Lett. 37(19), 4122-4124 (2012).

[6] Wiatrek, A., Preußler, S., Jamshidi, K., and Schneider, T., "Frequency domain aperture for the gain bandwidth reduction of stimulated Brillouin scattering," Opt. Lett. 37(5), 930-932 (2012).

[7] Preussler, S. and Schneider, T., "Stimulated Brillouin scattering gain bandwidth reduction and applications in microwave photonics and optical signal processing," Opt. Eng. 55(3), 031110 (2015).

[8] Zornoza, A., Sagues, M., and Loayssa, A., "Self-heterodyne detection for SNR improvement and distributed phase-shift measurements in BOTDA," J. Light. Technol. 30(8), 1066-1072 (2012).

[9] Soto, M. A., Le Floch, S., and Thévenaz, L., "Bipolar optical pulse coding for performance enhancement in BOTDA sensors," Opt. Express 21(14), 16390-16397 (2013).

[10] Jin, C., Guo, N., Feng, Y., Wang, L., Liang, H., Li, J., Li, Z., Yu, C., and Lu, C., "Scanning-free BOTDA based on ultra-fine digital optical frequency comb," Opt. Express 23(4), 5277-5284 (2015).

[11] Voskoboinik, A., Wang, J., Shamee, B., Nuccio, S. R., Zhang, L., Chitgarha, M., Willner, A. E., and Tur, M., "SBS-Based Fiber Optical Sensing Using Frequency-Domain Simultaneous Tone Interrogation," J. Light. Technol. 29(11), 1729-1735 (2011).

[12] Bernini, R., Minardo, A., and Zeni, L., "Dynamic strain measurement in optical fibers by stimulated Brillouin scattering.," Opt. Lett. 34(17), 2613-2615 (2009).

[13] Feng, C., Preussler, S., and Schneider, T., "Sharp tunable and additional noise-free optical filter based on Brillouin losses," Photonics Res. 6(2), 132-137 (2018).

[14] Marinelarena, J., Urricelqui, J., and Loayssa, A., "Enhancement of the Dynamic Range in Slope-Assisted Coherent Brillouin Optical Time-Domain Analysis Sensors," IEEE Photonics J. 9(3), 1-10 (2017).

[15] Urricelqui, J., Zornoza, A., Sagues, M., and Loayssa, A., "Dynamic BOTDA measurements based on Brillouin phase-shift and RF demodulation.," Opt. Express 20(24), 26942-26949 (2012). 